\title{
Multibiometric People Identification: A Self-tuning Architecture
}

\author{
Maria De Marsico ${ }^{2}$, Michele Nappi $^{1}$, and Daniel Riccio ${ }^{1}$ \\ 1 Universitá Degli Studi di Salerno, \\ via Ponte Don Melillo, 84084, Fisciano, Salerno, Italy \\ \{mnappi,driccio\}@unisa.it \\ 2 Universitá Degli Studi di Roma - La Sapienza, \\ via Salaria 113, 00198, Roma, Italy \\ demarsico@di.uniroma1.it
}

\begin{abstract}
Multibiometric systems can solve a number of problems of unimodal approaches. One source for such problems can be found in the lack of dynamic update of parameters, which does not allow current systems to adapt to changes in the working settings. They are generally calibrated once and for all, so that they are tuned and optimized with respect to standard conditions. In this work we propose an architecture where, for each single-biometry subsystem, parameters are dynamically optimized according to the behaviour of all the others. This is achieved by an additional component, the supervisor module, which analyzes the responses from all subsystems and modifies the degree of reliability required from each of them to accept the respective responses. The paper explores two integration architectures with different interconnection degree, demonstrating that a tight component interaction increases system accuracy and allows identifying unstable subsystems.
\end{abstract}

\section{Introduction}

Present systems generally rely on a single biometry. The main drawback is that they are singly vulnerable to possible attacks, as well as little robust with respect to a number of problems. Examples are acquisition errors, possibly due to bad hardware (e.g. a dirty sensor), as well as to an actual distortion of the biometric feature (e.g. a voice altered by a cold or a dimly lit face). In the present work we will consider the combination of iris-, face- and ear-based biometric identification systems. We chose these biometries because they are contact-less, fast and fairly reliable, as we will briefly discuss in the following.

The spatial patterns in the human iris make up a texture information which is highly distinctive of an individual, due to differences in the development of anatomical structures. In particular, biomedical literature [1] suggests that iris is as distinct as patterns of retinal blood vessels, but an iris image can be more easily obtained than a retina image. On the other hand, face is the most immediate characteristic through which we use to recognize a known person, so that many researchers' attention focuses on face recognition [2]. Moreover, people 
are especially used to be photographed and this makes this automatic recognition modality sufficiently accepted. Face recognition, however, raises a number of non-easy to solve issues, especially when dealing with pose, illumination or expression changes. This reduces reliability of related methods, that are still far from the recognition rates usually provided by DNA or fingerprints. Ear shows some advantages when compared to face. Iannarelli's in-depth medical investigations [3] demonstrated that also the ear meets the basic principle of distinctiveness, so that it is a biometry in every respect. The limited surface allows a faster processing than face, while the lack of expressive variations reduces intra-class variations. However, when the ear in not perfectly frontal with respect to the acquisition device, small variations can modify the obtained image even significantly. Moreover, occlusions due to earrings or hair might be more serious than an occlusion on a face, because of the limited exploitable surface.

A multibiometric system provides an effective alternative, as flaws of an individual system can be compensated by the availability of a higher number of cooperating biometries 4. In [5] System Response Reliability (SRR) indices have been introduced to evaluate the reliability of each response of single subsystems; responses are considered for fusion only if the corresponding SRR is higher than a given threshold th. This improves global system performance, but we argue that we can go even further by considering the "'history"' of the system. We assume the existence of a supervisor module exploiting not only single subsystems responses and their reliability, but also the final global response, to evaluate the overall system state and update reliability thresholds of single subsystems. Such module would allow overcoming the invariance of present multibiometric architectures, by implementing an algorithm converging to an optimal parameters configuration independently from the starting one. Each subsystem takes as input an image, pertaining to the corresponding biometry, and extracts salient features; the resulting feature vectors are used to compute similarity scores, to be inputted to the supervisor module, after a normalization step. As all the algorithms exploited for iris, face and ear rely on fractal theory, and in particular on PIFS (Partitioned Iterated Function System) [6], we briefly introduce some related core topics, and then describe the feature extraction procedures.

\section{The Integration Scheme}

We propose two multibiometric architectures with increasing complexity, a Parallel Protocol (PP) and a Supervised Protocol (SP). The latter significantly differs from the state of art in literature. The algorithm implemented by the recognition systems to classify the single biometry is always the same presented above. Therefore, the reported different performances can be unequivocally ascribed to the peculiar features of the different architectures. This consideration adds value to the experimental results in terms of consistency and readability.

\subsection{The Parallel Protocol}

In this first solution, PIFS are applied independently to each trait. The three corresponding subsystems work in parallel, each producing a list of subjects from 
its specific database of enrolled subjects (gallery); list elements include the ID of a database subject and a numeric score expressing its similarity with the input. Each list is ordered by such similarity, then the three lists are processed by a fusion module to obtain the global response. Two aspects have been especially investigated in designing this system: reliability margins and fusion policies.

Subsystems might not be equally reliable, e.g. due to the possibly different quality of input. An unreliable response can trigger a further check. A reliability measure is then crucial for fusion. Some solutions use margins, measuring the "risk" associated to a single subsystem response after observing its scores. Poh and Bengio [7] introduce a confidence margin based on False Acceptance Rate (FAR) and False Rejection Rate (FRR). Many responses are marked as reliable, as the margin relies on an estimate of the actual distribution of genuine/impostor subjects' scores. This might be inappropriate when very high security is required. Moreover, frequentist approaches assume that the scores of the testing and development sets always originate from similar distributions. We adopt the new System Response Reliability (SRR) index [5], based on a system/gallery dependent metric, and measuring the ability of separating genuine subjects from impostors on a single probe basis. SRR values always fall in the range $[0,1]$. Each subsystem $T_{k}$ computes, for each $s_{k, i}, \mathrm{i}=1, \ldots$, in its set of responses, a reliability measure $s r r_{k, i}$. Moreover, each subsystem $T_{k}$ is characterized by an estimated threshold $t h_{k}$, possibly updated over time 2, such that a response $s_{k, i}$ is considered as reliable only if $\operatorname{srr}_{k, i} \geq t h_{k}$.

Different policies exist for fusion. Veeramachaneni et al [8] investigate decision level fusion in a binary hypotheses-testing problem, and in particular the selection of the best subset from different fusion rules, to optimize FAR or FRR. Apart from the limitations of the approach, the interesting observations are that, for 2 classifiers fusion, AND and OR rules constitute a very important set (out of 16 possible ones), and that, as the number of classifiers increases, the optimal fusion rules are constructed from these two, giving better results than Averaged Sum Rule. This suggests that, despite the variety of fusion policies, we can assume that AND and OR generally represent a suitable choice. In our case, we have a system $S$ composed by 3 subsystems $T_{k},(k=1,2,3)$, processing iris, face and ear respectively, each exploiting a gallery $G_{k},(k=1,2,3)$ of images for the same set $H$ of enrolled identities. Notice that each subject can have more than one image in each single gallery. Our fusion rules also exploit the above System Response Reliability (SRR), since each $T_{k}$ returns a reliability value $s r_{k, i}$ for its $i$-th response. As for $O R$, the combined response is valid if at least one subsystem response is reliable; the system returns the identity retrieved by the subsystem with the higher reliability above the corresponding threshold. In $A N D$ policy, the combined response is valid only if all responses are reliable; the system returns the identity with the minimum weighted sum of distances from the probe, where weights are the reliability indexes. For sake of consistency, it is important to normalize distances before combining them.

Thresholds $t h_{k}$ can be fixed in advance, and remain fixed in time, or they can be computed and updated according to obtained responses. A compromise 
between the number of reliable responses and the system error rate must be obtained. Too high thresholds make the system too restrictive, with a low error rate but also a low number of acceptances, while too low ones might cancel the advantages of the reliability measure. Assume that $T_{k}$ has executed $M$ times, producing the set $\left\{s_{k, 1}, \ldots, s_{k, M}\right\}$ of responses. The corresponding reliability measures are combined in a set $R H=\left\{s r r_{k, 1}, \ldots, s r r_{k, M}\right\}$ containing the history of system behaviour (reliability history). The value to assign to $t h_{k}$ is strictly correlated to $\mathrm{RH}$ features, in particular to the mean and to the variance [5] of its elements. A high $\overline{R H}$ means that system responses are generally reliable so that its threshold can be proportionally high. On the other hand, the variance $\sigma^{2}[R H]$ measures the stability of $T_{k}$. The best situation is then when $R H$ elements have a high mean and a low variance, so that it is possible to fix a high value for $t h_{k}$. We can summarize the above observations in the formula:

$$
t h_{k}=\frac{\overline{R H^{2}}-\sigma^{2}[R H]}{R H},
$$

Our Parallel Protocol system, in particular $t h_{k}$ thresholds, has been adjusted according to a set of samples with similar features to those of the set used for identification tests; the two sets are different and disjoint.

\subsection{A Self-tuning Architecture: The Supervised Protocol}

A revisitation of the classical multibiometric schema takes to system self-tuning, with a much more flexible and robust architecture. The main limit of the architecture in Section 2.1 is that it does not seize the main advantage of exploiting information coming from other subsystems. Each component works independently and final results give no feedback for the overall system. Formula 1 is good for computing and updating the $t_{k}$ threshold for the reliability index of a single subsystem, according to its behaviour; however, it only accounts for that subsystem. On the contrary, assume the existence of a supervisor module (Supervised Protocol - SP) still exploiting single subsystem responses and their reliability to compute the final global response, but also using the latter to evaluate the overall system state and update its parameters (Fig. 1). Such module would implement an algorithm to update single thresholds also according to the behavior of the other subsystems, so converging to an optimal configuration independently from the starting $\left\{t h_{1}, t h_{2}, t h_{3}\right\}$ configuration. The algorithm distinguishes two cases:

Case I: More identities $I_{j}, j \in\{1,2, \ldots,|H|\}$ share the same maximum number of votes, e.g. when retrieved identities are all different with 1 vote each. If at least one $T_{k}$ in any such group has $s r r_{k}>t h_{k}$, the system returns the identity retrieved by the subsystem with the higher $s r r_{k}>t h_{k}$, and the response is marked as reliable, otherwise the response is marked as unreliable.

Case II: One identity $I_{j}$ gets more votes than the others. $I_{j}$ is returned and the response is marked as reliable. 
In both cases, if the response is reliable, each subsystem $T_{k}$ voting for the returned identity is rewarded by lowering its threshold th $h_{k}$ by an updating step $u s$, unless its current $s r r_{k}$ is already above its $t h_{k}$. Each other subsystem $T_{k}$ is penalized by increasing its threshold $t h_{k}$ by $u s$, unless its current $s r r_{k}$ is already below its $t h_{k}$. In this way the supervisor module lowers thresholds of subsystems voting in agreement, considering such behavior a confirmation of reliability, and increases thresholds of discordant ones, compensating possible distortions (local persistent distortions like lighting variations, dirt on lens).

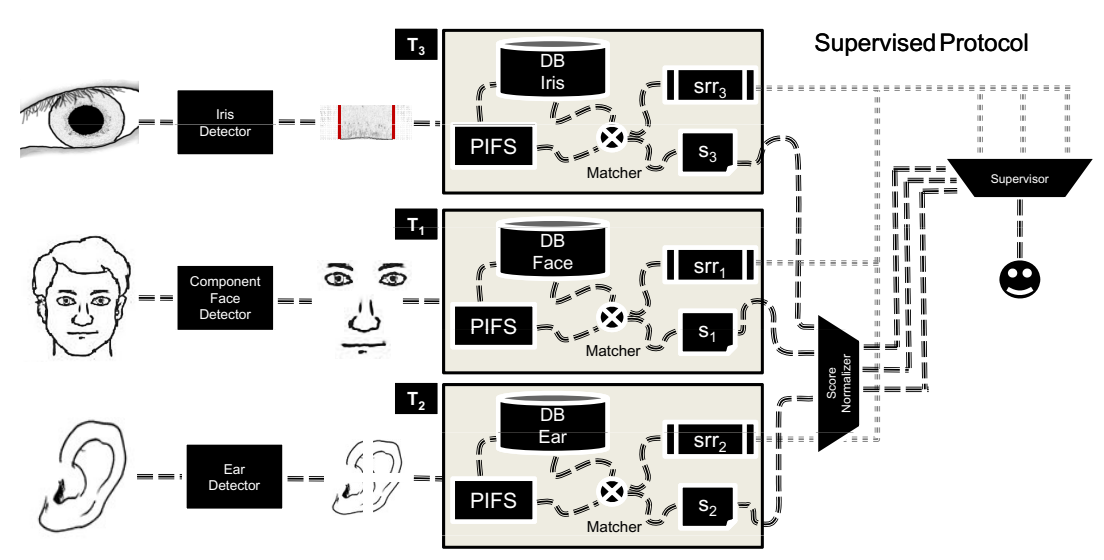

Fig. 1. The architecture of the supervised protocol

Such an architecture does not need an adjustment phase, since the system can start from a default configuration of its parameters and converge in any case towards an optimal one. The speed to reach such latter configuration is a significant system feature, so that it is important to define how to measure it. As we want to simulate the dynamic behavior of an online identification system, we assume that system time is beaten by performed recognition operations; we define a probe sequence $P=\left\{p_{1}, p_{2}, \ldots, p_{n}\right\}$ as a series of $n$ probes presented to the system, sharing the same acquisition characteristics (normal conditions, right light, earrings, dirty lens). A subsystem equilibrium state (steady state) is given by the consecutive instants when threshold fluctuations are lower than a fixed $\mu$, while convergence speed $\lambda_{k}$ of a subsystem is defined as the ratio between the total variation of its threshold between two consecutive steady states, and the number of instants needed to obtain such transition. Total system convergence speed is defined as the minimum speed among all its subsystems, i.e. $\lambda=\min _{k} \lambda_{k}, k \in\{1,2,3\}$.

\section{Experimental Results}

A suitable standard image database was chosen for each biometry: a) CASIA v 3.0 for iris [9], AR-Faces for face [10] and Notre-Dame for ear 11]. 100 different 
chimeric users were enrolled, by extracting the first 100 subjects from each database. In particular, 100 images of iris, face and ear (gallery set) were used for enrolling; further 100 images (tuning) for the same subjects, but distinct from those in the gallery, always in normal conditions, were used to compute Parallel Protocol (PP) parameters, namely $t h_{k}$ thresholds corresponding to reliability index $S R R$. Two different sets of images have been considered for testing (probe set). The first set probe- $I$ consists of normal conditions images different from those in the gallery and tuning sets; the second set probe- $I I$ contains the same images as probe- $I$ except that a gaussian filter was applied to them, with kernel $8 \times 8$ and variance $\sigma=3$, to simulate an out of focus situation. Images were manually segmented. As for irises, the center of the pupil, the minor radius and the major radius of the useful region were selected. Once extracted, such iris region was linearized and resized to a rectangle of $256 \times 360$ pixels. For each face, eyes, nose and mouth were located, while the useful region was resized to a square of $256 \times 256$ pixels. As for the ears, the upper left and the bottom right angle of the rectangle containing the ear were selected, in order to resize it to $200 \times 256$ pixels. Adopted measures for accuracy were Recognition Rate (RR), Equal Error Rate (EER) and Number of Reliable Responses (NRR). For each subsystem, the feature extraction process rely on PIFS (Partitioned Iterated Function System). PIFS are largely exploited in image compression and indexing, and they have also been investigated in the field of face and ear recognition [6].

The first experiment consisted in analyzing the performances of the different subsystems, which are summarized in Table 1. We can notice that, in normal conditions, the face shows the better performances, followed by iris and finally ear. This can be ascribed to two reasons: a) quality of iris images is lower than face images b) the exploited fractal techniques were especially optimized for face recognition. This is also confirmed by the fact that with "out of focus", when simulated through gaussian blur, performances of face recognition only drop of 0.07 , while those of iris and ear drop of 0.50 and 0.19 respectively. The inversion between ear and iris with blurred images might be due to the fact that the thin characteristic patterns in iris are much more affected by such operation.

\subsection{Comparisons among the Two Architectures}

In examining results, bear in mind that we are applying a dynamic architecture, while a static multibiometric scheme is usually adopted. We chose different test configurations, with probe sequences varying in the biometry or biometries bearing blurring distortion (e.g. normal iris, normal face and blurred ear). System performances are summarized in Table 2. To clarify the interpretation of the experiments, we notice that each of the selected image sets can be considered as a sequence $P$ of test images, sequentially submitted to the system. The initial thresholds configuration for SP is set at $\left\{t h_{1}=0.0, t h_{2}=0.0, t h_{3}=0.0\right.$, i.e. all responses are initially considered as reliable. The updating step us is fixed at 0.05. Table 2 shows that RR obtained with SP is comparable, or in some cases even better, than with PP, also obtaining a higher Number of Reliable Responses (NRR). More detailed observations pertaining the obtained RR and NRR can 
Table 1. Performance of single subsystems on probe- $I$ and probe-II

\begin{tabular}{ll|c|c}
\hline \multirow{2}{*}{\multicolumn{2}{c}{ SUBSETS }} & \multicolumn{2}{c}{ SINGLE SUBSYSTEMS } \\
\cline { 3 - 4 } \multicolumn{2}{c}{} & \multicolumn{2}{c}{ PERFORMANCES } \\
\cline { 3 - 4 } & & RR & EER \\
\hline \multirow{2}{*}{ IRIS } & NORMAL & 0.80 & 0.16 \\
& BLURRED & 0.30 & 0.22 \\
\hline \multirow{2}{*}{ FACE } & NORMAL & 0.97 & 0.03 \\
& BLURRED & 0.90 & 0.07 \\
\hline \multirow{2}{*}{ EAR } & NORMAL & 0.72 & 0.08 \\
& BLURRED & 0.53 & 0.14 \\
\hline \hline
\end{tabular}

also result from Table 2, NRR for PP never is never above 52, even guaranteeing a mean RR of 0.9967 . On the other hand, SP always succeeds in providing a mean NRR of 99.5 , with and RR of 0.97 in the worst case. This can be ascribed to the fact that PP implements a rigid $A N D$ policy, strongly limiting NRR to such cases when SRR indices of all voting systems are above the corresponding thresholds. On the contrary, SP shows a comparable mean value for RR of 0.985 , yet providing a mean NRR of 99.5. While in PP the drop in performances of one or more subsystems weighs on the global result by compromising the overall AND outcome, in SP the increment in the corresponding thresholds causes to rule out unreliable subsystems, so avoiding to decrease NRR though preserving an high RR. A further relevant observation is related to the way the two protocols set up th $h_{k}$ thresholds for reliability indexes. PP computes such thresholds once and for all using an equivalent tuning set, according to the formula (11), so that the face, which is characterized by an higher average reliability (high mean), presents an high value for its threshold $t h_{2}$. On the contrary, SP, which also takes into account what happens in the other two subsystems, rewards the face recognizer with a lower threshold. In other words, it implements an even opposite philosophy, taking to better performances. If a subsystem is generally in agreement with the others, we can accept also "less" reliable responses from it. Finally, notice how, in SP, a decrease in input quality in the face subsystem causes a corresponding increase in the reliability threshold. This does not undermine the correctness of the formula (1), but demonstrates its inadequacy in the case of multibiometric systems.

\subsection{Further Experiments on the Supervised Protocol}

The first experiment aimed at highlighting how the threshold configuration towards which the system converges is independent from the starting one, and how the threshold updating algorithm is stable. We considered 100 probe sequences of 1000 images randomly extracted among the 100 of sets probe- $I$ (face and ear) and probe-II (iris). For each system run, the initial values for thresholds were randomly chosen (all values being equally probable) in the interval $[0,1]$. Results of this experiment are reported in Fig. 2, showing that curves generated by the different probe sequences tend to always concentrate in a relatively small final interval. This confirms the convergence of the updating procedure. We can 
Table 2. Performance comparison on different input configurations: Parallel Protocol $(\mathrm{PP})$ and Supervised Protocol (SP)

\begin{tabular}{|c|c|c|c|c|c|c|c|c|c|c|}
\hline \multirow{3}{*}{\multicolumn{3}{|c|}{ CONFIGURATIONS }} & \multicolumn{8}{|c|}{$\begin{array}{c}\text { MULTI-BIOMETRIC ARCHITECTURES } \\
\end{array}$} \\
\hline & & & \multicolumn{4}{|c|}{ PARALLEL PROTOCOL (PP) } & \multicolumn{4}{|c|}{ SUPERVISED PROTOCOL (SP) } \\
\hline & & & \multirow{2}{*}{$\begin{array}{c}\text { PERF. } \\
1.00\end{array}$} & \multirow{2}{*}{$\mathrm{th}_{1}$} & \multirow{4}{*}{$\begin{array}{l}\mathrm{th}_{2} \\
0.15\end{array}$} & \multirow{4}{*}{$\begin{array}{l}\mathrm{th}_{3} \\
\\
0.02\end{array}$} & \multirow{2}{*}{$\begin{array}{c}\text { PERF. } \\
1.00\end{array}$} & \multirow{4}{*}{$\begin{array}{l}\mathrm{th}_{1} \\
0.04\end{array}$} & & \multirow{2}{*}{$\mathrm{th}_{3}$} \\
\hline IRIS & NORM & $\mathrm{RR}$ & & & & & & & & \\
\hline FACE & NORM & EER & 0.00 & 0.06 & & & - & & 0.02 & 0.10 \\
\hline EAR & NORM & NRR & 43 & & & & 100 & & & \\
\hline IRIS & NORM & $\mathrm{RR}$ & 1.00 & \multirow{3}{*}{0.06} & \multirow{3}{*}{0.15} & \multirow{3}{*}{0.16} & 1.00 & \multirow{3}{*}{0.10} & \multirow{3}{*}{0.04} & \multirow{3}{*}{0.62} \\
\hline FACE & NORM & EER & 0.01 & & & & - & & & \\
\hline EAR & BLUR & NRR & 36 & & & & 100 & & & \\
\hline IRIS & NORM & RR & 1.00 & \multirow{3}{*}{0.06} & \multirow{3}{*}{0.09} & \multirow{3}{*}{0.02} & 0.99 & \multirow{3}{*}{0.06} & \multirow{3}{*}{0.13} & \multirow{3}{*}{0.06} \\
\hline FACE & BLUR & EER & 0.00 & & & & - & & & \\
\hline EAR & NORM & NRR & 45 & & & & 100 & & & \\
\hline IRIS & BLUR & RR & 1.00 & \multirow{3}{*}{0.44} & \multirow{3}{*}{0.15} & \multirow{3}{*}{0.02} & 0.97 & \multirow{3}{*}{0.84} & \multirow{3}{*}{0.02} & \multirow{3}{*}{0.12} \\
\hline FACE & NORM & EER & 0.00 & & & & - & & & \\
\hline EAR & NORM & NRR & 52 & & & & 99 & & & \\
\hline IRIS & NORM & RR & 1.00 & \multirow{3}{*}{0.06} & \multirow{3}{*}{0.09} & \multirow{3}{*}{0.16} & 0.98 & & & \\
\hline FACE & BLUR & EER & 0.04 & & & & - & 0.06 & 0.10 & 0.40 \\
\hline EAR & BLUR & NRR & 39 & & & & 100 & & & \\
\hline IRIS & BLUR & RR & 0.98 & & & & 0.97 & & & \\
\hline FACE & NORM & EER & 0.02 & 0.44 & 0.15 & 0.16 & - & 0.94 & 0.02 & 0.64 \\
\hline EAR & BLUR & NRR & 41 & & & & 98 & & & \\
\hline
\end{tabular}

observe that the threshold $t h_{2}$ (face) tends to assume lower values than $t h_{1}$ and $t h_{3}$ (iris and ear). Notice that the curves pertaining to subsystems iris and ear span a larger interval; this fact underlines that, as they are less reliable on average, they are more easily influenced by the other subsystems. The darker line (in black) in Fig. 2 is the mean value of the 100 computed curves and represents the mean trend for thresholds variation. For each threshold, the mean $(\{0.92$, $0.02,0.12\})$ and variance $(\{0.08,0.02,0.09\})$ of values in the 100 iterations were computed; the same for RR (mean: 0.981, variance: 0.004) and NRR (mean: 980, variance: 1.09). Fig. 3 shows the thresholds variation over a probe sequence composed of 5 subsequences of 200 images each. For each subsequence, we simulate a malfunctioning for one or more subsystems by randomly extracting their input images from probe-II. The other subsystems will randomly extract input images from probe-I. In Fig. 3. we show this by using "N" for normal images (probe- $I$ ) and "B" for blurred ones (probe- $I I)$. Each subsequence is composed of randomly extracted images from sets probe-I (Normal Images - N) and probe-II (Blurred Images - B). Notice that for the first sequence the thresholds $t h_{1} \mathrm{e}$ $t h_{3}$ reach higher values than $t h_{2}$, consistently with the fact that on set probe-I face presents the higher reliability. The configuration dramatically changes in the third sequence, in which iris images come from set probe-II, where the presence of blur lowers reliability of subsystem $T_{1}$. In sequence 4 , the blur effect on input ear images lowers performances of $T_{3}$ subsystem, while the only one to remain more reliable is $T_{1}$. Fig. 3 shows some examples of the computation of the subsystems convergence speed, namely for $T_{3}$, and of the identification of equilibrium states with the corresponding value of $\mu$, for $T_{1}$; it is also to notice a case when all the subsystems are in equilibrium, so that we can speak about system equilibrium. 

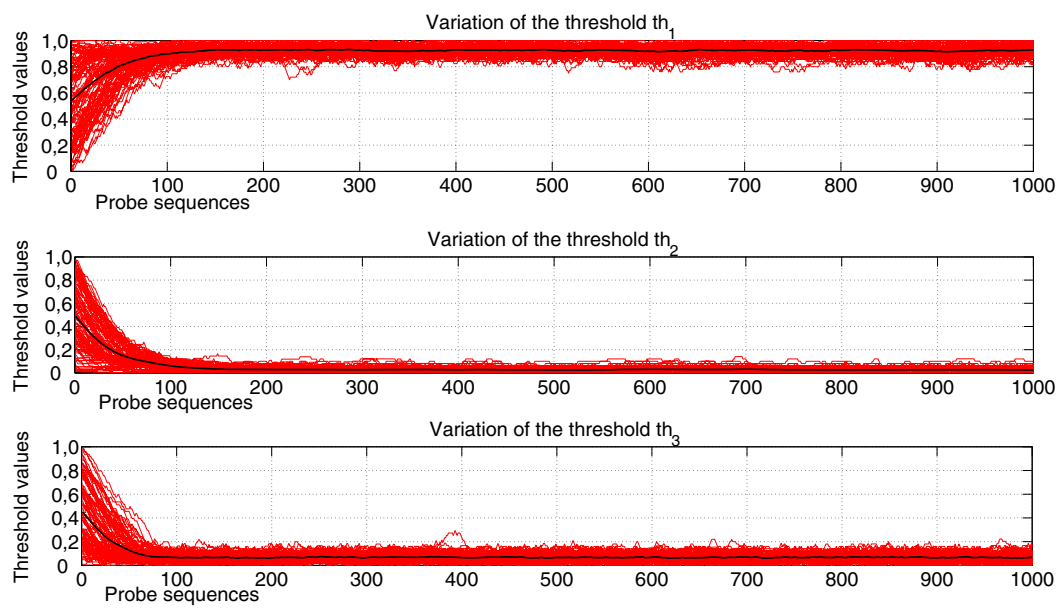

Fig. 2. Variation of thresholds of subsystems $T_{1}, T_{2}$ and $T_{3}$ for 100 probe sequences of 1000 images of 100 subjects according to the initial thresholds configuration ( $\left\{t h_{1}\right.$, $\left.\left.t h_{2}, t h_{3}\right\}\right)$; the black curve represents the mean trend

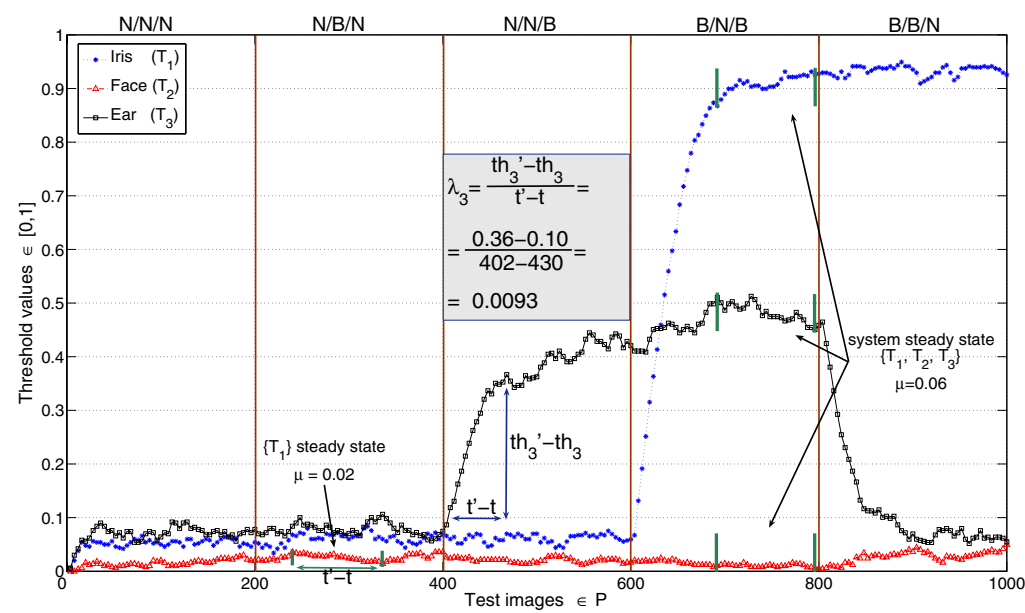

Fig. 3. Example of thresholds variation $\left(T_{1} / T_{2} / T_{3}\right)$ over a probe sequence of 1000 images from set probe-I (Normal Images - N) and probe-II (Blurred Images - B)

\section{Conclusions}

Multibiometric systems, though resolving a number of problems of singlebiometry ones, are generally somehow penalized from the invariance of their parameters, making them unable to adapt to changes in the conditions of their working environment. In this paper, we propose an architecture aiming at overcoming such limitation, through the introduction of a supervisor module. Such 
additional component collects information from the different subsystems and exploits them to modify the internal conditions (parameters) of each subsystem, aiming at improving the global response. This work opens a line to further investigations, where aspects such as a deeper action of the supervisor module on the internal subsystems state, or gallery updating, represent potential integrations to the architecture presented herein.

\section{Acknowledgements}

This work has been partially supported by the Provincia di Salerno Administration, Italy.

\section{References}

1. Adler, F.H.: Physiology of the eye: Clinical Application, 4th edn. The C.V. Mosby Company, London (1965)

2. Abate, A.F., Nappi, M., Riccio, D., Sabatino, G.: 2d and 3d face recognition: A survey. Pattern Recogn. Lett. 28(14), 1885-1906 (2007)

3. Iannarelli, A.: Ear identification. In: Calif, Paramont Publishing Fremont (eds.) Forensic Identification Series, pp. 67-74 (1989)

4. Ross, A., Jain, A.K., Qian, J.-Z.: Information fusion in biometrics. In: Bigun, J., Smeraldi, F. (eds.) AVBPA 2001. LNCS, vol. 2091, pp. 354-359. Springer, Heidelberg (2001)

5. Riccio, D., De Marsico, M., Abate, A.F., Nappi, M.: Data normalization and fusion in multibiometric systems. In: International Conference on Distributed Multimedia Systems, DMS 2007, pp. 87-92 (2007)

6. Riccio, D., Tortora, G., Abate, A.F., Nappi, M.: Rbs: A robust bimodal system for face recognition. International Journal of Software Engineering and Knowledge Engineering 17(4), 497-514 (2007)

7. Poh, N., Bengio, S.: Improving fusion with margin-derived confidence in biometric authentication tasks. In: Kanade, T., Jain, A., Ratha, N.K. (eds.) AVBPA 2005. LNCS, vol. 3546, pp. 474-483. Springer, Heidelberg (2005)

8. Veeramachaneni, K., Osadciw, L., Varshney, P.K.: An adaptive multimodal biometric management algorithm. IEEE Transaction on Systems, Man, and CyberneticsPart C: Applications and Reviews 35, 344-356 (2005)

9. Chinese Academy of Sciences. Note on casia-iris v.3. Website (2008), http://www.cbsr.ia.ac.cnIrisDatabase.htm

10. Martinez, A.M.: Recognizing imprecisely localized, partially occluded, and expression variant faces from a single sample per class. IEEE Transaction on Pattern Analisys and Machine Intelligence 24, 748-763 (2002)

11. Flynn, P.: Notre dame ear database, und principal investigator: Professor patrick flynn, computer science and engineering, university of notre dame, notre dame. Website, 2007-01-07 (2007) 